\title{
Research Paper \\ Prediction of Alexithymia Based on Abnormal Personality Dimension
}

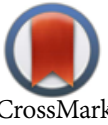

\author{
*Reza Abdi ${ }^{1}$, Gholamreza Chalabianloo ${ }^{1}$, Afsaneh Joorbonyan ${ }^{2}$
}

1- PhD of Psychology, Assistant Professor, Department of Psychology, Faculty of Education and Psychology, Azarbaijan Shahid Madani University, Tabriz, Iran 2- PhD Student, Department of Psychology, Faculty of Education and Psychology, University of Tabriz, Tabriz, Iran.

Citation: Abdi R, Chalabianloo Gh, Joorbonyan A. [Prediction of Alexithymia Based on Abnormal Personality Dimension (Persian)]. Iranian Journal of Psychiatry and Clinical Psychology. 2016; 22(3):222-229. https://doi.org/10.18869/acadpub.ijpcp.22.3.222

: https://doi.org/10.18869/acadpub.ijpcp.22.3.222

Received: 24 Jan. 2016 Accepted: 31 May 2016

Keywords:

Alexithymia, Abnormal personality dimensions, Diagnostic and Statistical Manual of Mental Disorders

\section{ABSTRACT}

Objectives Alexithymia, as a personality trait, causes malfunctioning in individuals in three areas of recognizing emotions, describing emotions, and defective objective thinking. The present study aims to investigate the role of these three abnormal personality dimensions introduced in the 5th volume of the Diagnostic and Statistical Guide for Mental Disorders in the prediction of aspects of alexithymia. Based on previous works, we have hypothesized that there is a correlation between abnormal personality dimension and alexithymia dimensions.

Methods In order to test the proposed hypothesis, a sample of high-school students of Ramsar County $(\mathrm{N}=250)$ were evaluated using the Personality Inventory for DSM-5 (PID-5) and Toronto Alexithymia Scale. Data were analyzed using SPSS18 and by the method of enter regressions.

Results The results indicated that there was a significant relationship between the inability to identify emotion and negative affect $(r=0.28)$, disinhibition $(r=0.20)$ and psychoticism $(r=0.16)$. It was also revealed that there was a significant positive relationship between the description of emotion and negative affect $(r=0.19)$, detachment $(r=0.14)$, disinhibition $(r=0.16)$ and psychoticism $(r=0.27)$ and between objective thinking and all abnormal personality dimensions $(P<0.01)$. Moreover, these personality dimensions can serve as a useful factor for predicting alexithymia.

Conclusion According to the obtained correlations between alexithymia and abnormal personality dimension, it is necessary that the comorbidity of these two variables be considered in the treatment of personality and emotional disorder.

* Corresponding Author:

Reza Abdi, PhD

Address: Department of Psychology, Faculty of Education and Psychology, Azarbaijan Shahid Madani University, Tabriz, Iran.

Tel: +98 (41) 35558179

E-mail: r.abdi@azaruniv.ac.ir 


\title{
ييشبينى ابعاد ناتويى هيجانى بر اساس مدل ابعاد نابهنجار شخصيت
}

\author{
"زضا عبدى'، غلامرضا جلبيانلو'، افسانه جوربنيان’ \\ 1- دكتراى روانشناسى، استاديار، كروه روانشناسى، دانشكده علوم تربيتى و روانشناسى، دانشكاه شهيد ملنى آذربايجان، تبريزء ايران.

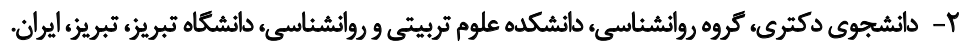

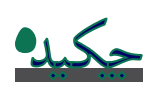

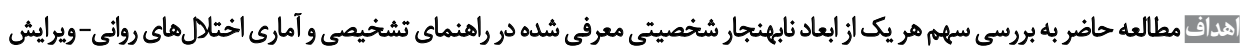

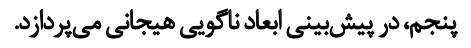

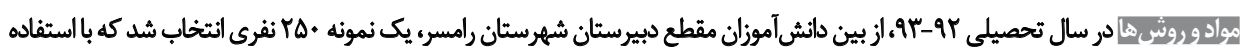

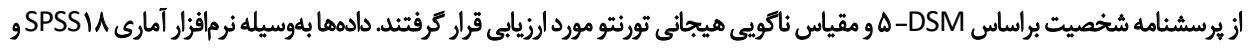

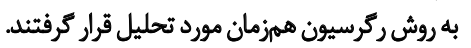

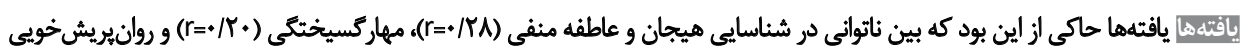

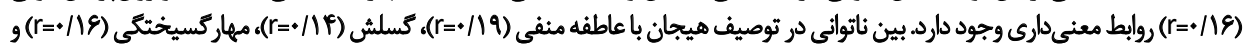

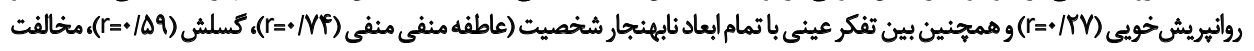

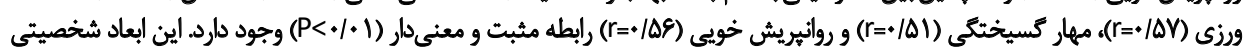

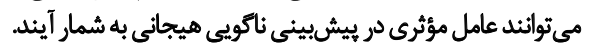

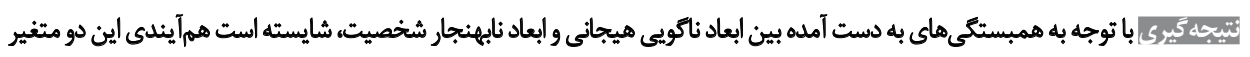

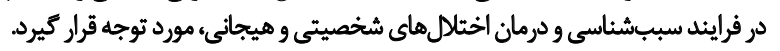

تاريخ دريافت: P بهيمن Pa

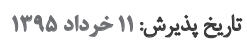
: sojlg
نائويى هيجاني، ابعاد

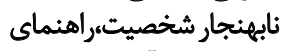 تشخيصى و آمارى اختلال هاى روانى

رفع آنها نيز، به درمانهاى جسمانى رو مى آورند [ه].

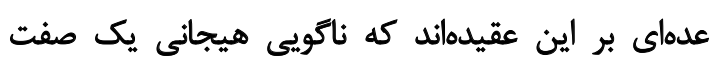

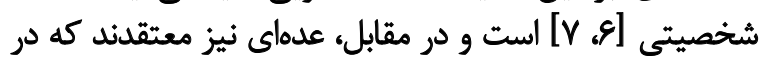

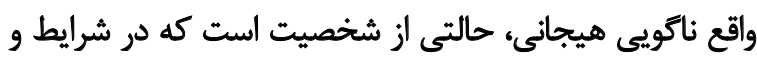

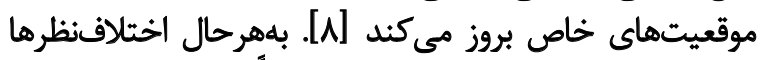

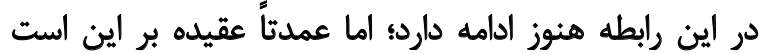

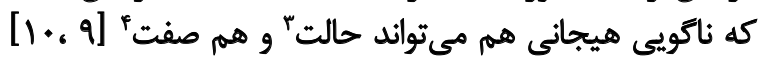

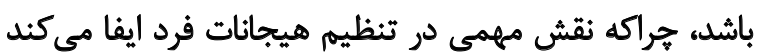

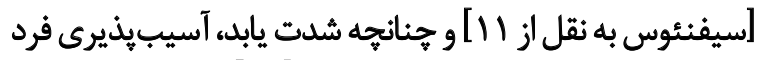

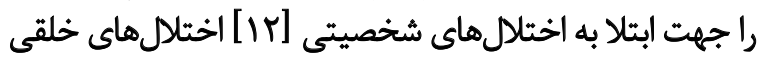

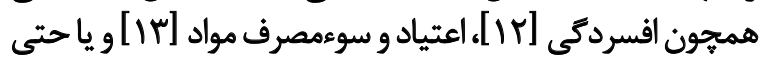

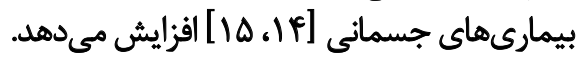

\section{State}

4. Trate dales واره نائويى هيجاني' اولين بار توسط سيفنئوسج، براي

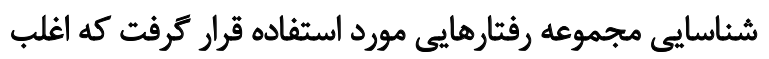

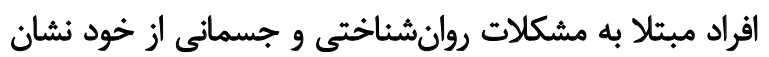

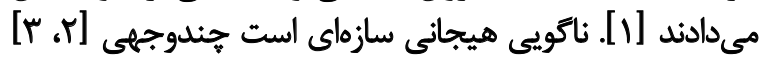

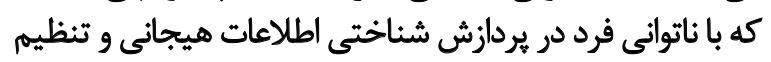

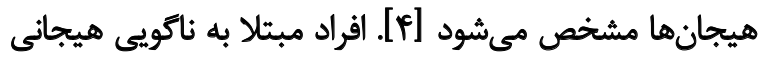

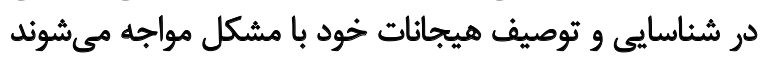

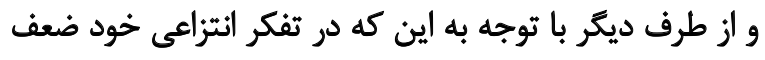

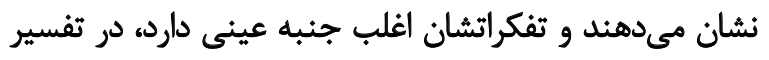

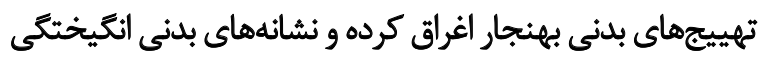
هيجان را به صورت نامناسب ثفنسير مي كنيند [f]

\section{Alexithymia}

2. Sifneos 
شخصيت بر اساس يُنجمين ويراست راهنماي تشخيصى و آمارى

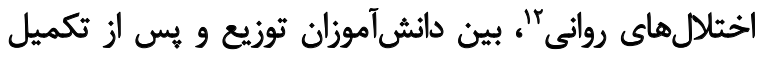

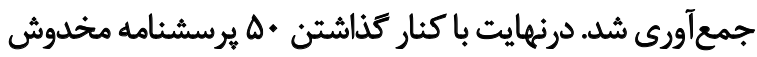

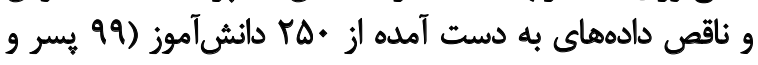

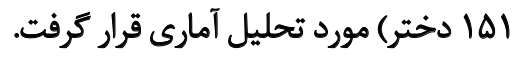

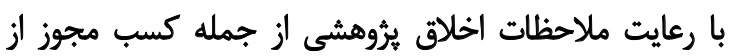

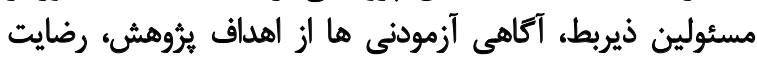

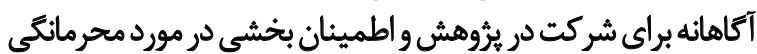

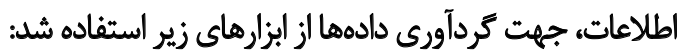

\section{مقياس ثائويى هيجائي تورئتو}

اين مقياس در سال 1999 توسط بكبي، باركر و ثايلور طراحي

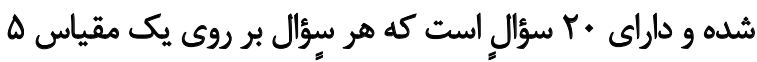

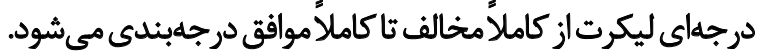

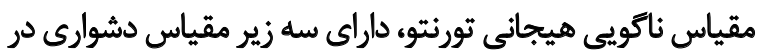

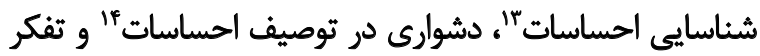

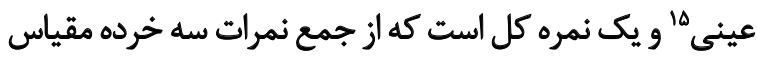

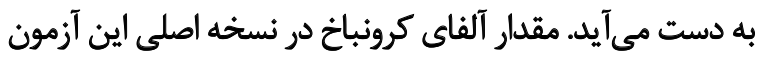

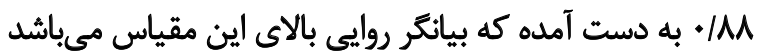

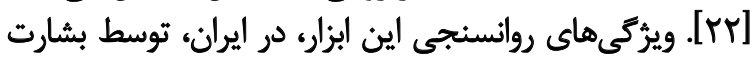

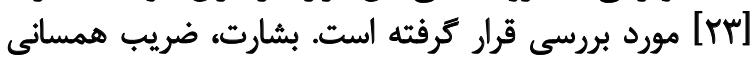

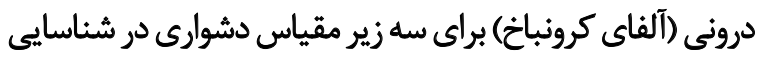

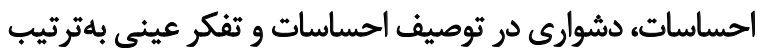

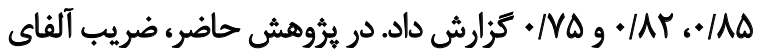

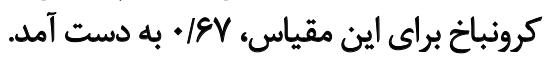

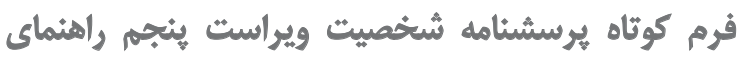
تشخيصى و أمارى اختلال هاي روائي (BF-Q-PID)

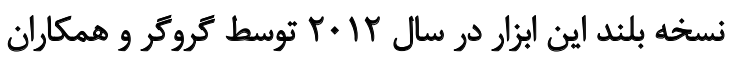

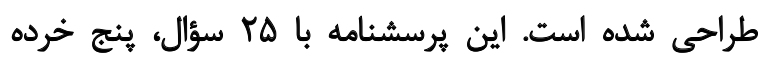

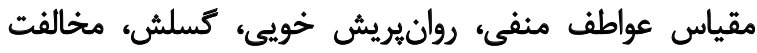

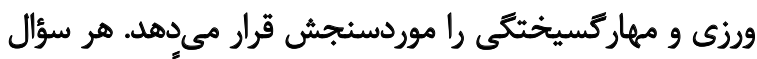

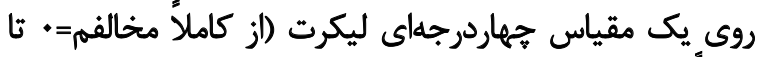

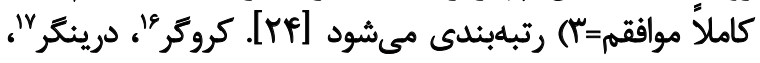

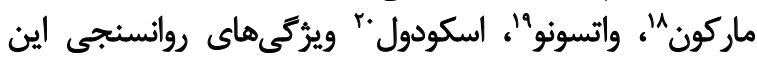

12. Personality Inventory for DSM-5-Brief Form (PID-5-BF)

13. Diffculty identifying feelings

14. Difficulty describing feelings

15. Externally oriented thinking

16. Krueger

17. Derringer

18. Markon

19. Watson

20. Skodol
از جمله عواملى كه مي توانند در بروز ناكويى هيجاني مؤثر باشند،

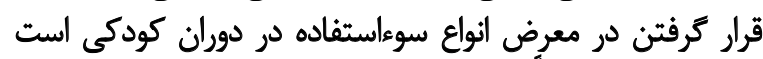

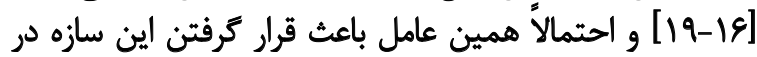

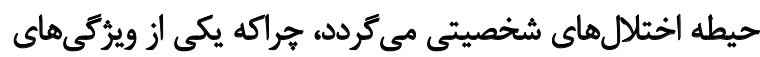

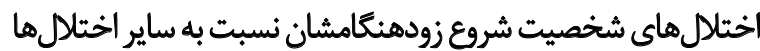

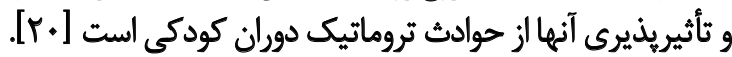

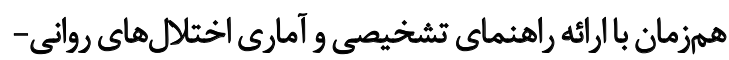

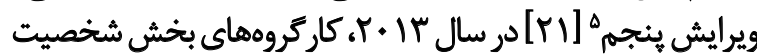

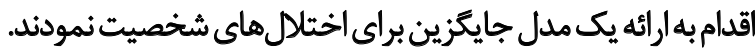

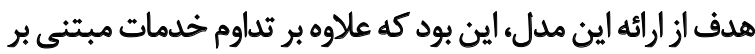

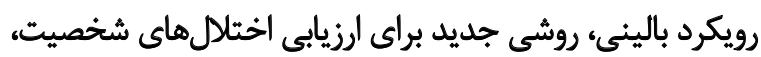

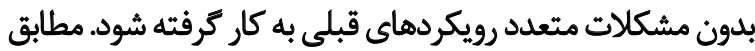

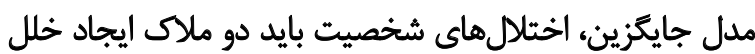

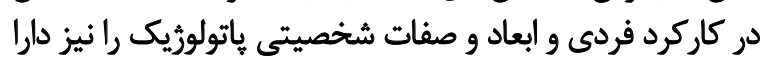

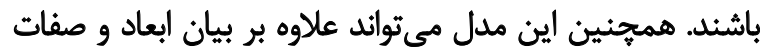

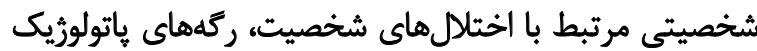

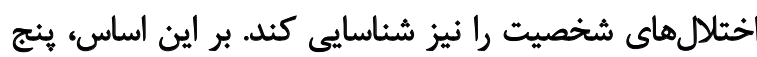

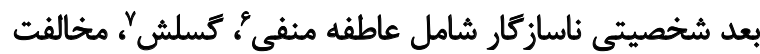

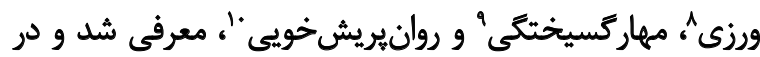

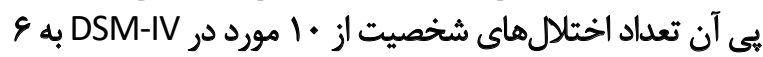

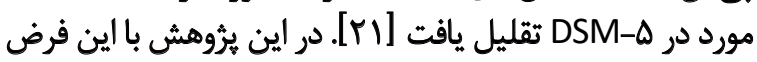

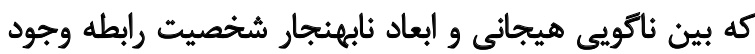

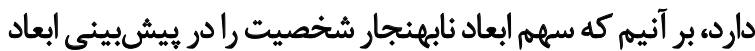

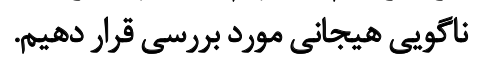
روش

طرح يُروهشى حاضر از نوع توصيفى و همبستكى است.

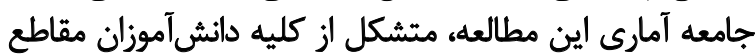

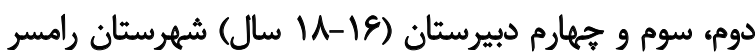

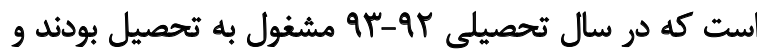

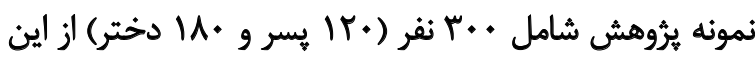

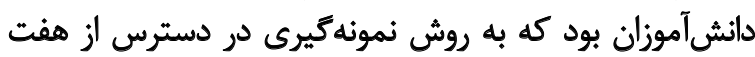

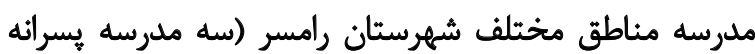

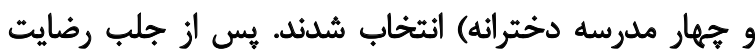

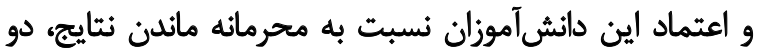

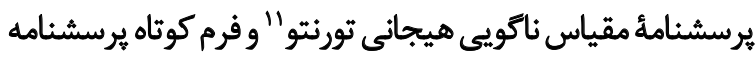

5. Diagnostic and Statistical Manual of Mental Disorders- Fifth Edition (DSM-5)

6. Negative affect

7. Detachment

8. Antagonism

9. Disinhibition

10. Psychoticism

11. Toronto Alexithymia Scale 
19/T سال بوده و همجئين ميانكين ابعاد عاطفه منفى، كسلش،

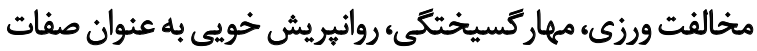

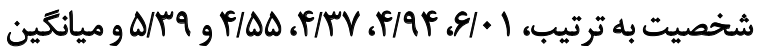

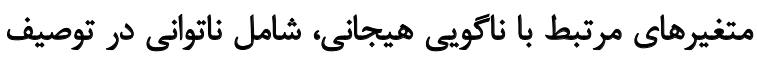

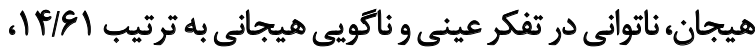

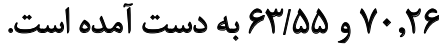

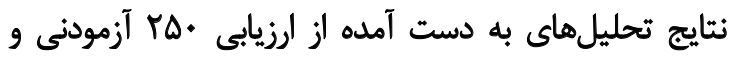

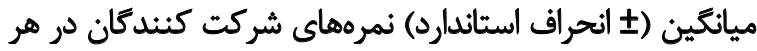

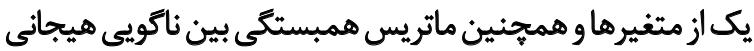

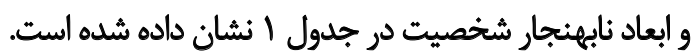

مطابق اطلاعات ارائه شده در جدول ا، بين ناتوانى در

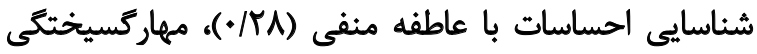

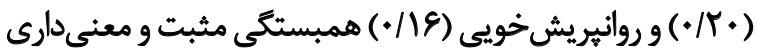

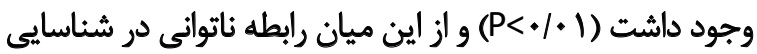

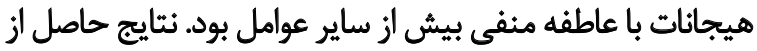

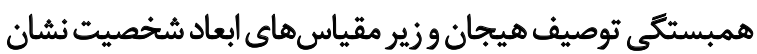

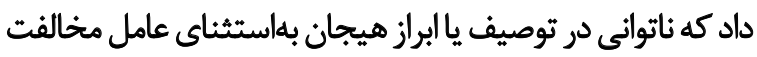

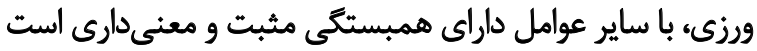

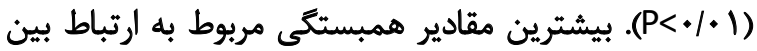

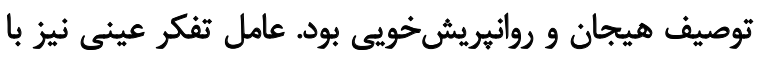

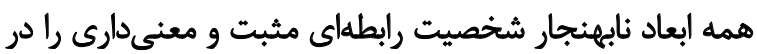

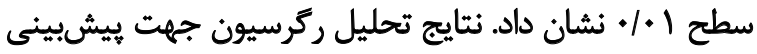

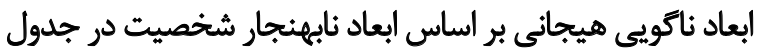
بارائه كرديده است.
آزمون رادر نمونههاى جمعيت عادى و افرادى كه در جستوجوى روان

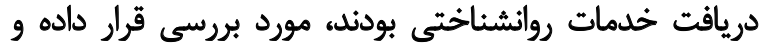

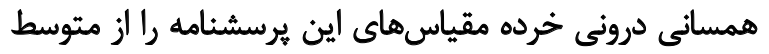

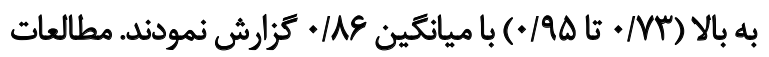

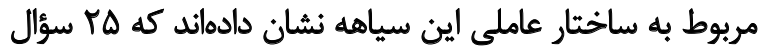

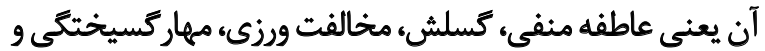

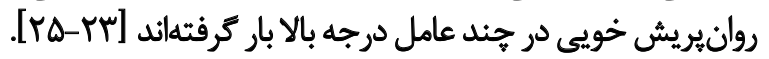

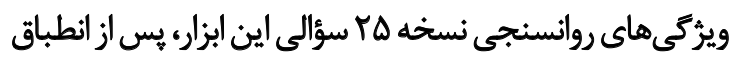

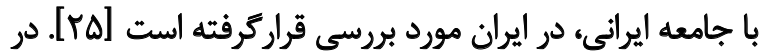

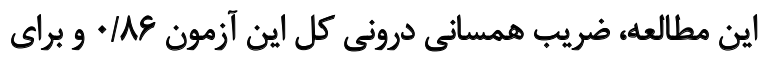

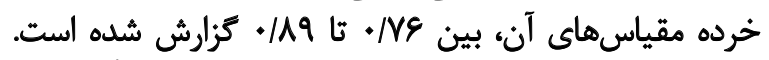

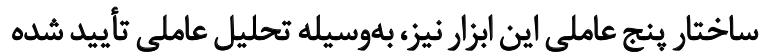

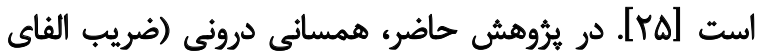
كرونباخ)، س الم/ • به دست آمدو حاضر،

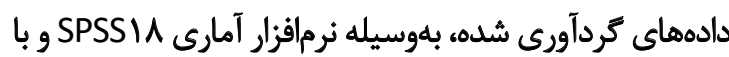

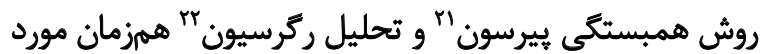
تجزيه و تحليل قرار كرفت.

ياثتهها

يافتههاى مطالعه حاضر حاصل تحليل اطلاعات به دست

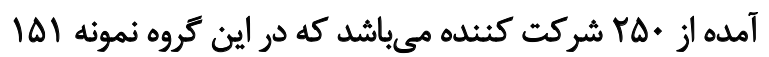

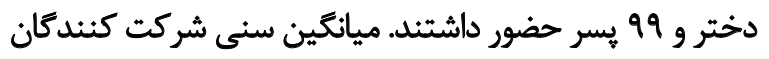

21. Pearson correlation

22. Regression analysis

جدول ا. ماتريس همبستكى ناكويى هيجانى و ابعاد نابهنجار شخصيت.

\begin{tabular}{|c|c|c|c|c|c|c|c|c|c|}
\hline \multicolumn{8}{|c|}{ ضرايب همبستكى (r) } & \multirow{2}{*}{ 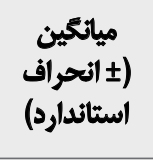 } & \multirow[b]{2}{*}{ متغيرها } \\
\hline تفيني & توصيف & شئاسايى & روانيريش & مهارتكسيختكى. & مخالفت & كَّلش & منفِْيه & & \\
\hline & & & & & & & 1 & $8 /+1(r / *+)$ & عاطفه منفى \\
\hline & & & & & & 1 & $\cdot / r V$ & $P / Q P(Y / \cdot Y)$ & كسلش \\
\hline & & & & & 1 &.$/ M$ & $\cdot / r$ & $P / T V(Y / A \Delta)$ & مخالفت ورزى \\
\hline & & & & 1 &.$/ K E$ & זוז/. &.$/ 41$ & $F / \Delta \Delta(T / Y A)$ & مهاركسيختكى \\
\hline & & & 1 & $\cdot / 4 F$ & $\cdot / \mu \Lambda$ &.$/ N A$ &.$/ 48$ & $\Delta / T q(T / A F)$ & روانيريشخويى \\
\hline & & 1 & $+/ 180$ & $\cdot / r * *$ & $.1 \cdot 1$ & $+\infty+\infty$ &.$/ r \lambda^{* *}$ & $T r / M T\left(r / q_{0}\right)$ & شناسايى هيجان \\
\hline & 1 & ./N1 & $+/ T V^{* *}$ &.$/ C^{* *}$ & $\%$ & $* / N+*$ &.$/ 19 * *$ & $|f / g|(r / r q)$ & توصيف هيجان \\
\hline 1 &.$/ 18$ & T &.$/ \Delta Q^{\circ}$ & $\cdot \mid \Delta)^{\infty}$ & $\cdot / \Delta V^{e+}$ & $\cdot / \Delta q^{* \bullet}$ & $\cdot / M=0$ & $r E / V \cdot(F / M F)$ & تفكر عينى \\
\hline$\cdot / M I$ & .189 & $\cdot M$ & . $/ \pi^{n+*}$ & $\cdot(Y)^{* *}$ & .1 .8 & $.1 . Y$ & ./rq** & $\varepsilon^{\prime} / \Delta \Delta(\vartheta / \cdots)$ & ناكويى هيجانى \\
\hline
\end{tabular}


جدول r. خلاصه نتايج تحليل ركرسيون جهت بيشبينى ابعاد ناكويى هيجانى بر اساس ابعاد نابهنجار شخصيت.

\begin{tabular}{|c|c|c|c|c|c|c|c|c|}
\hline Sig. & $\mathbf{F}$ & $\mathbf{R}$ & $\mathbf{R}^{r}$ & Sig. & $\mathbf{T}$ & بتا & مثغير ملاى & مثغير ييشبين \\
\hline$\%$ & a/re &.$/ 4$ & .1 .9 & $\%$ & $r / \Delta)$ &.$/ \pi f$ & شناسايع هيجان & عاطفه منفى \\
\hline$\%$ & $\Delta / N^{r}$ & I & .1 .9 & $\begin{array}{l}. \% \mu \\
.\end{array}$ & $\begin{array}{l}-r / T V \\
r / F q\end{array}$ & $\begin{array}{l}-. / N F \\
. / M F\end{array}$ & توصيف هيجان & مواثتيريشت ورزى \\
\hline$\%$ & $F / 9 \Delta$ & $+/ 79$ & $.1 \cdot 1$ & $\begin{array}{l}\% \\
1 \% \\
1 \% \\
1 \%\end{array}$ & $\begin{array}{l}10 / 99 \\
1 . / 4 T \\
N Q T \\
R / T \Delta\end{array}$ & $\begin{array}{l}. / 4 . \\
. / 41 \\
. / 4 V \\
. / 1\end{array}$ & تفكر عينى & 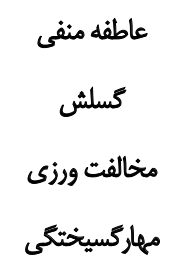 \\
\hline$\%$ & $V / .9$ &.$/ \pi$ &.$/ 11$ & $\begin{array}{l}. . \\
.4\end{array}$ & $\begin{array}{l}r / T 1 \\
T / I I\end{array}$ & ./RT & ناكويى هيجانى & روانيريش خويى منفى \\
\hline
\end{tabular}

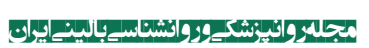

اين ويرُكى ناكويى هيجانى را به عنوان حلقه اتصال افسردگى و و

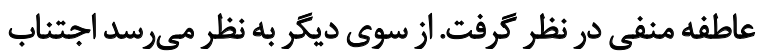

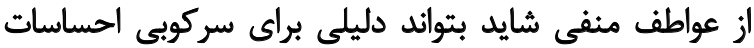
ناخوشايند و در نتيجه نارسايى در توصيف اين هيجانات باني باشيد

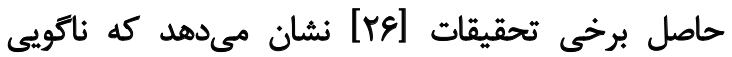

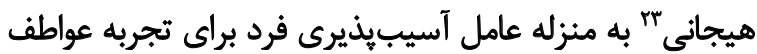

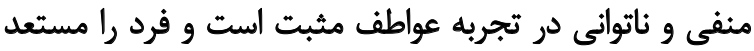

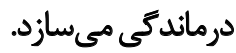

در يرؤهش حاضر، بين مهاركسيختكى و ناتوانى در شناسائي

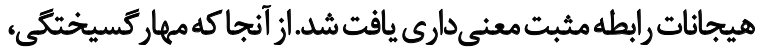

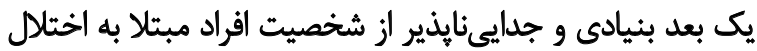

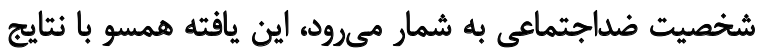

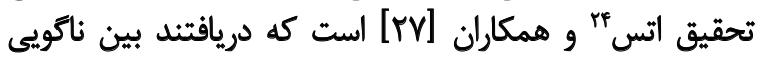
هيجانى و اختلال شخصيت ضداجتماعى رابطه مثبت وجود دارد.

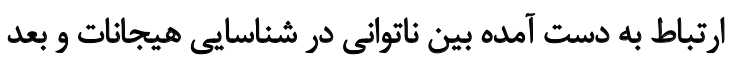

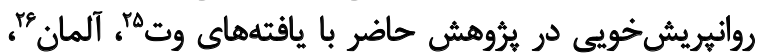

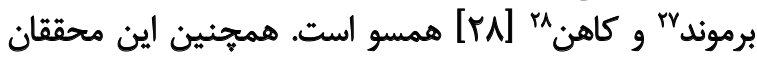

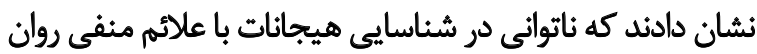

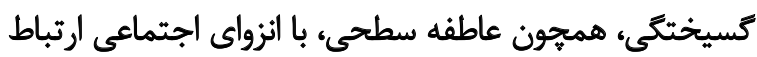

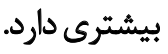

23. Emotional

24. Ates

25. Wout

26. Aleman

27. Bermond

28. Kahn
همانطور كه جدول r نشان مي دهد، بعد شخصيتى عاطفه

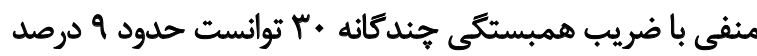

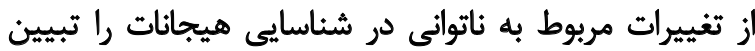

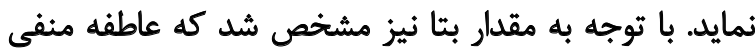

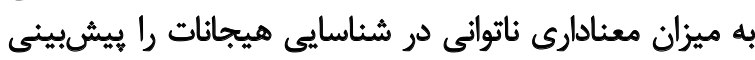
مى كند. در رابطه با توصيف هيجان نيان نيز نتايج نشان ديان داد كه ابعاد

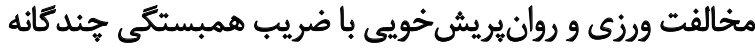

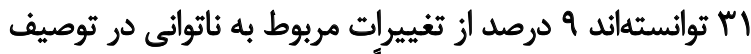

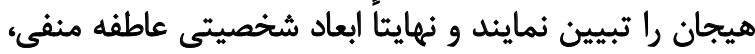

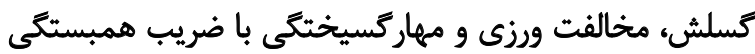

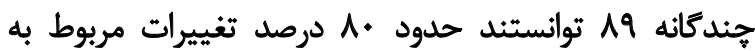

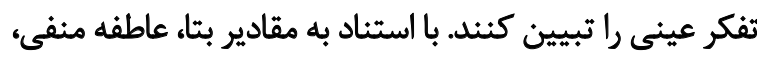

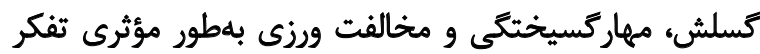

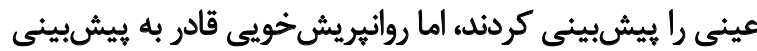

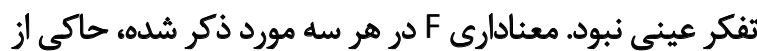

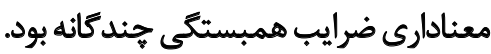
ث̊?

يُروهش حاضر با هدف بررسى سهم هر يك از ابعاد نابهنجار

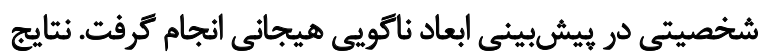

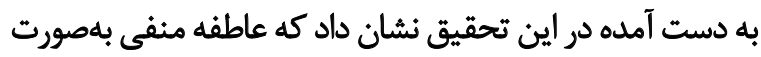

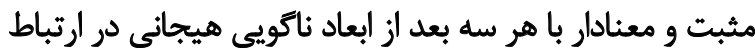

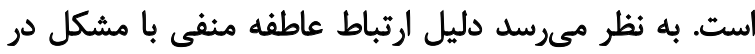

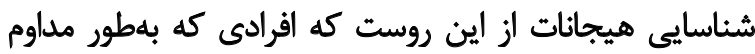

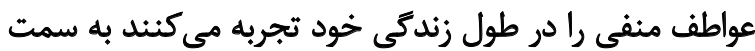

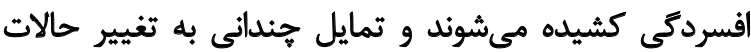

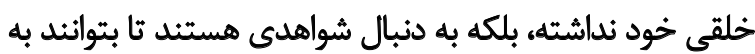

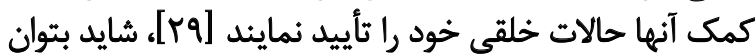


ناكويى هيجانى است كه با همأ ابعاد نابهنجار شخصيت ارتباط

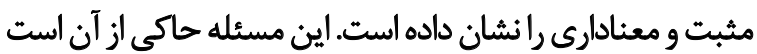

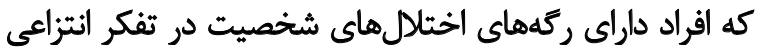

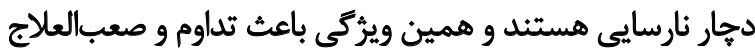
شدن اين طيف از اختلال هاى مي مكردد.

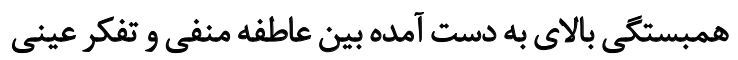

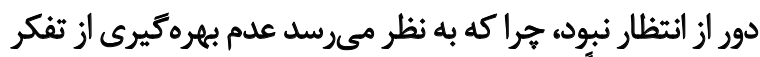

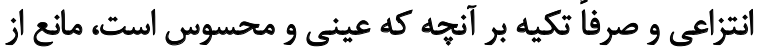

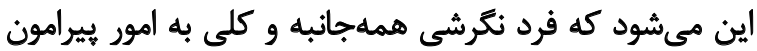

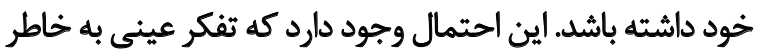

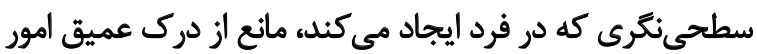

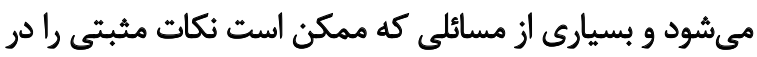
دل خود نهفته باشند را سوءتعبير نمايد.

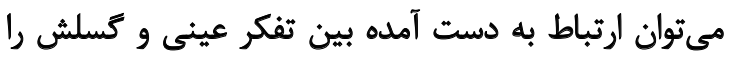

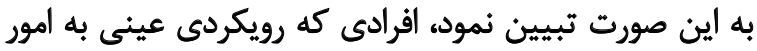

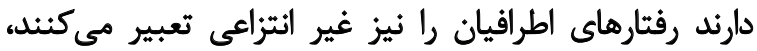

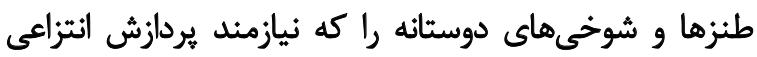

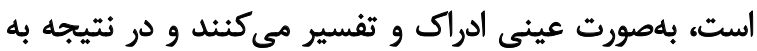

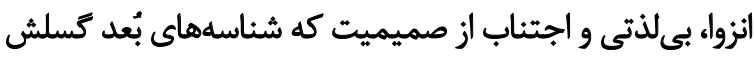

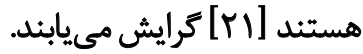

ضعف در تفكر انتزاعى و صرفأ تكيه بر تفكرات عينى، ممكن

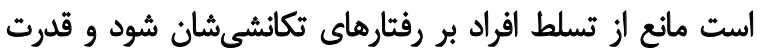

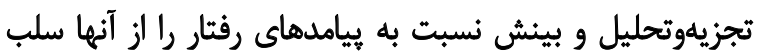

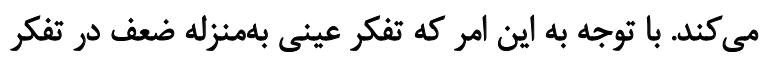

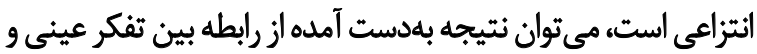

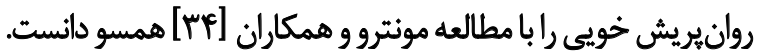

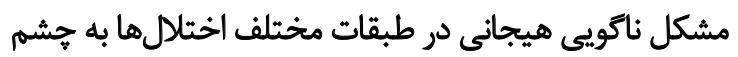

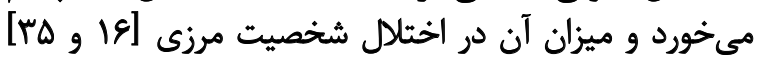

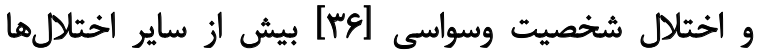

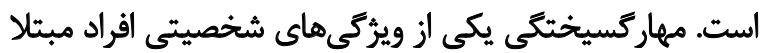

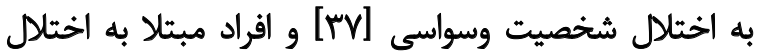

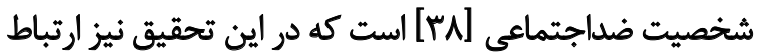
اين بعد با ابعاد ناتويى هيجانى مشاهده شد.

ترؤهش حاضر نشان داد كه بين ناكويى هيجاني (ناتوانى در

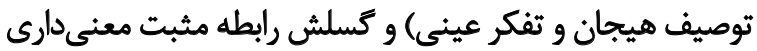

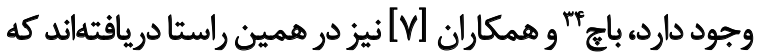

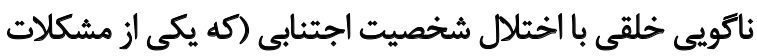

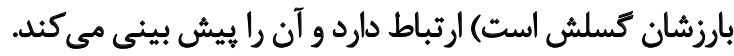

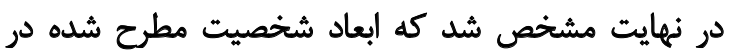

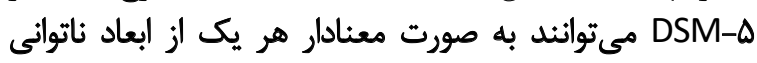

كسانى كه از نظر روان كسيختكى نمرات بالايى كسب

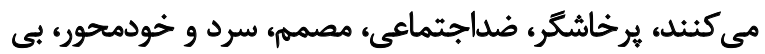

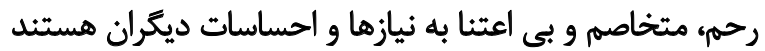

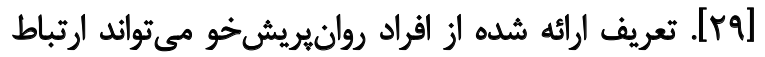

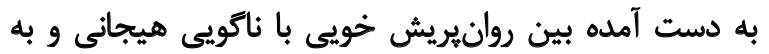

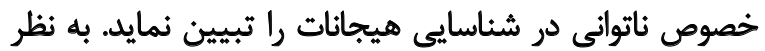

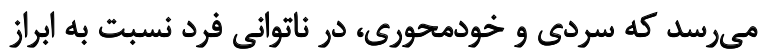

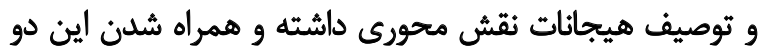

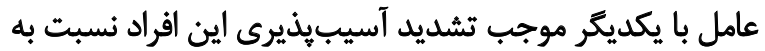
اختلال هاى شخصيت مى كردد.

همجنين يُؤهش حاضر همسو با يافتههاى راس كلافل،

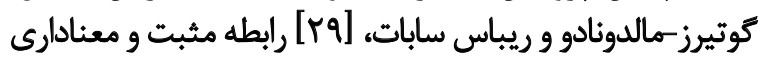

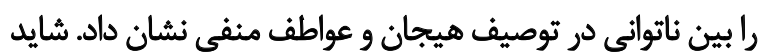

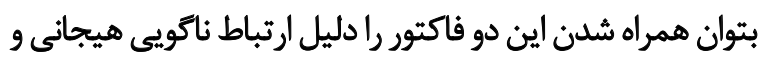

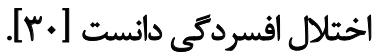

ارتباط مثبت بين ناتوانى در ابراز احساسات و كسلش نشان

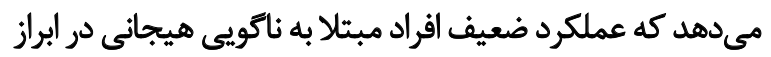

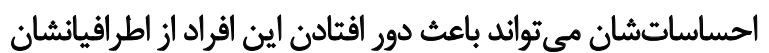

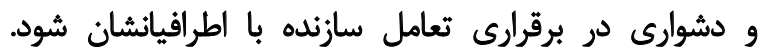

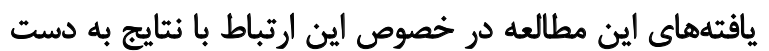

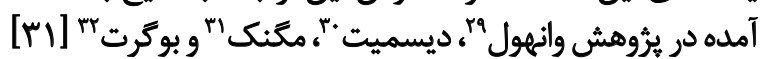

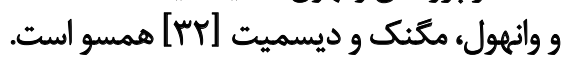

در اين مطالعه ارتباط معنادارى بين ناتوانى در ابراز هيجانات

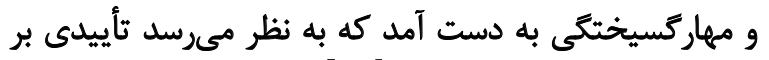

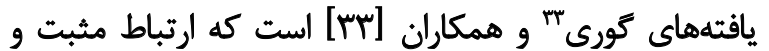

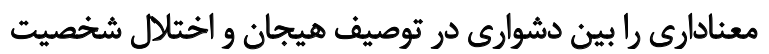

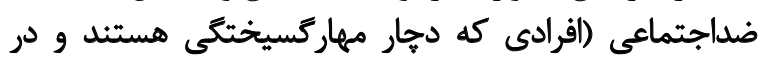
كنترل تكانههاى خود مشكل دارند) به دست آوردمانداند.

نتايج برؤوهش جارى، ارتباط مثبت و معنادارى را بين ناتوانى

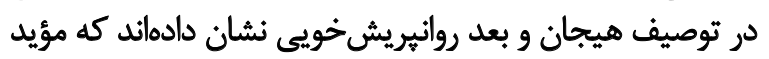

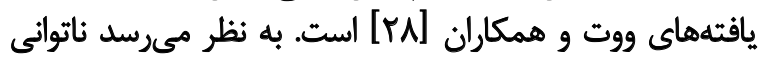

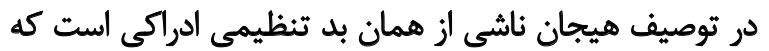

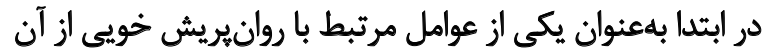

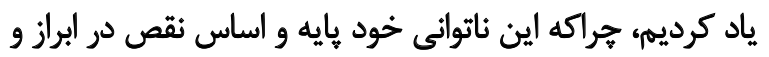

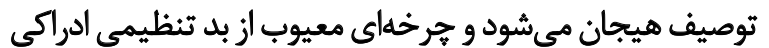
و ناثوانى در ابراز هيجان ران هيديد مى آورد. مطابق يافتههاى اين مطالعه تفكر عينى تنها بعد از ابعاد 


\section{References}

[1] Humphreys TP, Wood LM, Parker JDA. Alexithymia and Satisfaction in Intimate Relationships. Pers Individ Dif. 2009; 46: 43-47.

[2] Bermond B, Oosterveld P, Vorst HCM. Measures of Personality and Social Psychological Constructs. Academic Press; 2015. p. 13-14.

[3] Taylor GJ. Bagby RM. An Overview of the Alexithymia Construct. In: Bar-On R, Parker JD, Editors. The Handbook of Emotional Intelligence: Theory, Development, Assessment, and Application at Home, School and In the Workplace. 1sted. San Francisco: Jossey-Bass; 2000. p.263-76.

[4] Taylor GJ, Parker JD, Bagby RM, Acklin MW. Alexithymia and Somatic Complaints in Psychiatric Out-Patients. J Psychosom Res. 1992; 36: 417-424.

[5] Assar Kashani H, Roshan R, Khalaj A, Mohammadi J. A Study on the Alexithymia and Body Image in the Obese, Over-Weight and Normal Waight Subjects. Health Psychol. 2012; 1: 70-80. (Persian)

[6] Wise TN, Mann LS, Shay L. Alexithymia and the Five-Factor Model of Personality. Compr Psychiatry. 1992; 33: 147-151.

[7] Bach M, Zwaan MD, Ackard D, Nutzinger D, Mitchell JE. Alexithymia: Relationship to Personality Disorders. Compr Psychiatry. 1994; 35: 239-243

[8] Marchesi C, Giaracuni G, Paraggio C, Ossola P, Tonna M. PreMorbid Alexithymia in Panic Disorder: A Cohort Study. J Psychiatr Res. 2013; 215: 141-145.

[9] De Haan HA, Hein AJV, Wijdeveld TGM, Buitelaar JK, De Jong CAJ. Alexithymia in patients with substance use disorders: State or trait? J Psychosom Res. 2014; 216: 137-145.

[10] Salminen JK, Saarioi S, Toikka T, Kauhanen J, Aarela E. Alexithymia Behaves As A Personality Trait Over A 5-Year Period In Finnish General Population. J Psychosom Res. 2006; 61: 275-278.

[11] O’Driscoll `D, Laing J, Mason O. Cognitive Emotion Regulation Strategies, Alexithymia and Dissociation in Schizophrenia, a Review and Meta-Analysis. Clin Psychol Rev. 2014; 34: 482- 495.

[12] Coolidge FL, Estey AJ, Segal DL, Marle PD. Are Alexithymia And Schizoid Personality Disorder Synonymous Diagnoses? Compr Psychiatry. 2013; 54: 141-148.

[13] De Haan H, Joosten E, Wijdeveld T, Boswinkel P, Palen JVD, Jong CD. Alexithymia Is Not A Stable Personality Trait In Patients With Substance Use Disorders. J Psychiatr Res. 2012; 198:123-129.

[14] Pontone S, Marianetti M, Mina C, Pontone P. Personality of Patients With Upper Gastrointestinal Symptoms: Alexithymia, Anxiety, Depression And Coping Style Investigation In A Preliminary Study. J Gastroenterol. 2011; 140: 468.

[15] Grabe HJ, Schwahn Ch, Barnow S, Spitzer C, John U, Freyberger HJ, Schminke Ulf, Felix S Völzke H. Aexithymia, Hypertension, and Subclinical Atherosclerosis in the General Population. J Psychosom Res. 2010; 68: 139-147.

[16] Evren C, Cinar O, Evren B. Relationship of Alexithymia and Dissociation with Severity of Borderline Personality Features in Male Substance-Dependent Inpatients. Compr Psychiatry. 2012; 53: 854-859.
در شناسايي هيجان، ناتواني در توصيف هيجان و تفكر عيني و

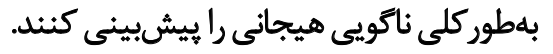

ملاحظه شد كه وجود عاطفه منفي در همه ابعاد ناكويى هيجاني

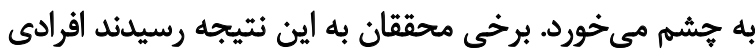

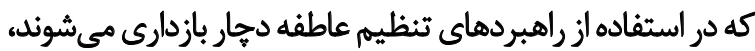

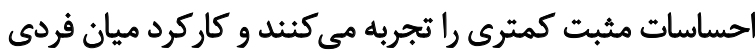

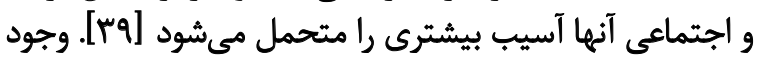

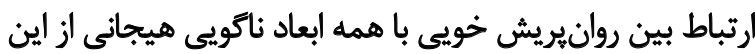

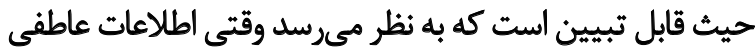

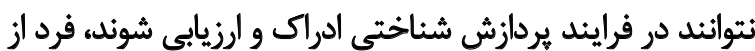

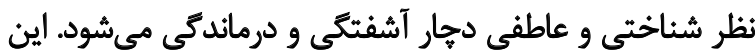

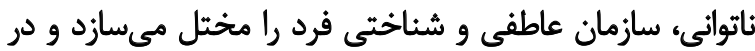

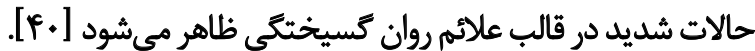

$$
\text { S.Satow }
$$

بنا بر آنجهه مطرح شده محققان و متخصصان تشخيص و درمان

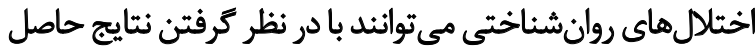

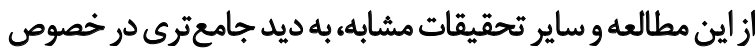

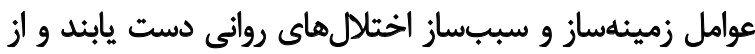

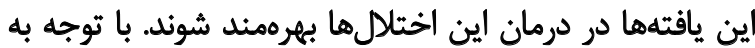

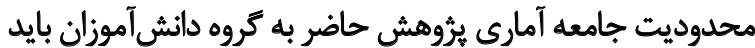

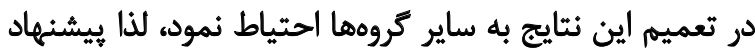

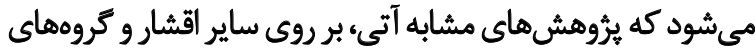

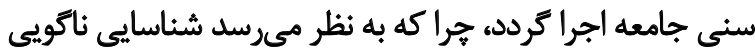

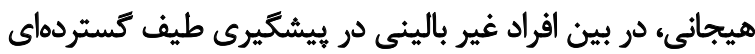

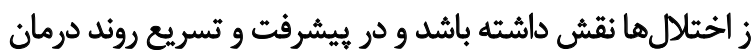

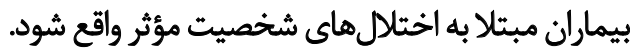

$$
\text { سياسكزإ) }
$$

بدينوسيله از كليه افرادى كه در تكميل اين يروهش ما رايارى

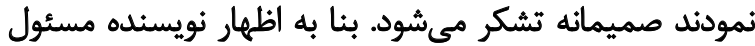

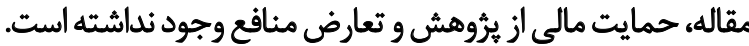


[17] Zlotnick C, Tracie Shea M, Teri P, Simpson E, Costello E, Begin A. The Relationship Between Dissociative Symptoms, Alexithymia, Impulsivity, Sexual Abuse, And Self-Mutilation. Compr Psychiatry. 1996; 37: 12-16.

[18] Berenbaum H. Childhood Abuse, Alexithymia and Personality Disorder. J Psychosom Res. 1996; 41: 585-595.

[19] Hund AR, Espelage DL. Childhood Emotional Abuse And Disordered Eating Among Undergraduate Females:M Mediating Influence Of Alexithymia And Distress. Child Abuse Negl. 2006; 30: 393-407.

[20] Zhang TH, Chow a, Wang LL, Dai YF, Xiao X. Role of childhood traumatic experience in personality disorders in China. Compr Psychiatry. 2012; 53: 829-836.

[21] American Psychiatric Association. Diagnostic and Statistical Manual of Mental Disorders (DSM-5). Washington, DC: Author; 2013.

[22] Bagby RM, Taylor GJ, Parker JDA. The Twenty-Item Toronto Alexithymia Scale: II. Convergent, Discriminant, And Concurrent Validity. J Psychosom Res.1994; 38: 33-40.

[23] Besharat MA. Alexithymia and defensive style. J Public Fundam Health. 2007; 3: 181-190.

[24] Krueger RF, Derringer J, Markon KE, Watson D, Skodol AE. Initial construction of a maladaptive personality trait model and inventory for DSM-5. Psychol Med. 2012; 42: 1879-1890.

[25] Abdi R, Chalabianloo GhR. A preliminary study of adaptation and psychometric properties of the short form version of the Diagnostic and Statistical Manual of Mental Disorders of adult personality inventory fifth edition of DSM-5 (PID-5-BF). J New Psychol Res. In Press. (Persian)

[26] De Gucht V, Fischler B, Heiser W. Neuroticism, Alexithymia, Negative Affect, And Positive Affect As Determinants Of Medically Unexplained Symptoms. Pers Individ Dif. 2004; 36: 16551667.

[27] Ates MA, Semiz UB, Semiz A, Algul A, Ebrinc C, Basoglu S, Iyisoy $\mathrm{O}$, Gecici $\mathrm{O}$, Cetin M. Alexithymia and Aggression in $\mathrm{Pa}$ tients with Antisocial Personality Disorder. Eur Psychiat. 2008; 23: S. 91

[28] Wout MV, Aleman A, Bermond B, Kahn RS. No words for feelings: alexithymia in schizophrenia patients and first- degree relatives. Compr Psychiatry. 2007; 48: 27-33.

[29] Rus-Calafell M, Gutiérrez-Maldonado J, Ribas-Sabaté J. Affectivity, alexithymia and psychopathology in a psychotic sample. Pers Individ Dif. 2014. 60: S24-S47.

[30] Bagby RM, Taylor GJ, Ryan D. Toronto alexithymia scale: relationship with personality and psychopathology measures. Psychother Psychosom. 1986; 45: 207-215.

[31] Vanheule S, Desmet M, Meganck R, Bogaerts S. Alexithymia and interpersonal problems. J Clin Psychol. 2007; 63: 109-117.

[32] Vanheule S, Meganck R, Desmet M. Alexithymia, social relations andmental processing: An explorative study.Psychiat Res. 2011; 190: 49-51.

[33] Gori A, Craparo G, Iraci Sareri G, Caretti V, Gianninia M, Meringolo P. Antisocial and psychopathic personalities in a sample of addicted subjects:Differences in psychological resources, symptoms, alexithymia and impulsivity. Compr Psychiatry. 2014; 55: 1580-1586.

[34] Montoro CI, del Paso GA, Duschek S. Alexithymia in fibromyalgia syndrome. Pers Individ Dif. 2016 Nov 30;102:170-9.

[35] Stepp SD, Scott LN, Morse JQ, Nolf KA, Hallquist MN, Pilkonis PA. Emotion Dysregulation as A Maintenance Factor of Borderline Personality Disorder Features. Compr Psychiatry. 2014; 55: 657- 666.

[36] Narimani M, Vahidi Z, Abolqasemi A. Comparison Alexithymia, Impulsivity and Activation and Inhibition of the Students with Symptoms of Obsessive-Compulsive and Paranoid Personality Disorder with Normal Individuals. J Clin Psychol. 2013 Jul 15;5(2):55-65. [Persian]

[37] Wu KD, Clark LA, Watson D. Relations between ObsessiveCompulsive Disorder and Personality: Beyond Axis I-Axis II Comorbidity. J Anxiety Disord. 2006; 20: 695- 717

[38] Kendler Kenneth S, Aggen Steven H, Patrick Christopher J. A Multivariate Twin Study of the DSM-IV Criteria for Antisocial Personality Disorder. J Biol Phys. 2012; 71: 247-253.

[39] Gross JJ, John OP. Individual Differences in Two Emotion Regulation Processes: Implications for Affect, Relationships, and Well-Being. J Pers Soc Psychol. 2003; 85: 348-362.

[40] Abolghasemi A, Bahari M, Narimani M, Zahed A, mood and alexithymia compare in Psychosis with positive and negative symptoms. J Psychol Sci.2011; 19: 1-10. [Persian] 